\title{
Automated Quantitation of the Posterior Scleral Flattening and Optic Nerve Protrusion by MRI in Idiopathic Intracranial Hypertension
}

N. Alperin, A.M. Bagci, B.L. Lam, and E. Sklar

\begin{abstract}
BACKGROUND AND PURPOSE: Subjective determination of the posterior sclera flattening and optic nerve protrusion in MRI is challenging because of the 3D nature of the globe morphology. This study aims to develop and compare quantitative measures of globe flattening and optic nerve protrusion with subjective rating, and assess relationships with papilledema grade and intraocular and CSF pressures.
\end{abstract}

MATERIALS AND METHODS: Data of 34 globes from 7 overweight female patients with idiopathic intracranial hypertension and 6 ageand weight-matched healthy female control subjects were assessed, as well as a subcohort of 4 of the patients with idiopathic intracranial hypertension who underwent follow-up MR imaging 2 weeks after lumbar puncture and initiation of treatment with acetazolamide. MR imaging examination included a 3D CISS sequence on 1.5T and 3T scanners with 0.6-mm isotropic resolution. Subjective ratings of globe flattening were obtained by experienced and inexperienced readers. Quantitative measures of globe flattening, nerve protrusion, and maximal deformation were derived by use of a 2D map of the distances from the globe center to the posterior wall.

RESULTS: Contingency coefficients for globe flattening agreements with subjective rating by the experienced and inexperienced readers were 0.72 and 0.56 , respectively. Mean values of the 3 deformation measures were significantly poorer in the idiopathic intracranial hypertension group, with nerve protrusion demonstrating the strongest difference $(P=.0002)$. Nerve protrusion was most strongly associated with papilledema grade with a contingency coefficient of $0.74(P=.01)$, whereas globe flattening was negatively correlated with intraocular pressure $(R=-0.75, P<.0001)$. Maximal deformation was negatively associated with CSF opening pressure $(R=-0.86, P=$ $.0001)$. After treatment, only the changes in nerve protrusion and maximal deformation were significant.

CONCLUSIONS: Automated measures of globe deformation improve reliability over subjective rating. Of the 2 globe deformation measures, nerve protrusion had the strongest predictive value for papilledema grade and had the highest sensitivity for assessment of treatment efficacy in idiopathic intracranial hypertension.

ABBREVIATIONS: $I \mathrm{OP}=$ intraocular pressure; $\mathrm{IH}=$ intracranial hypertension; $I \mathrm{IH}=$ idiopathic intracranial hypertension; $\mathrm{LP}=$ lumbar puncture; $I \mathrm{CP}=$ intracranial pressure; $\mathrm{NP}=$ nerve protrusion; $\mathrm{GF}=$ globe flatness; $\mathrm{MD}=$ maximal deformation

$\mathbf{F}_{\text {of }}$ attening of the posterior sclera and inward protrusion of the optic nerve head are recognized MR imaging markers of intracranial hypertension $(\mathrm{IH}){ }^{1-5}$ These findings have been reported primarily in idiopathic intracranial hypertension $(\mathrm{IIH})^{1-3}$ but also in secondary $\mathrm{IH}$, including sinus thrombosis and nonthrombotic cases of

Received February 5, 2013; accepted after revision March 19, 2013.

From the Department of Radiology (N.A., A.M.B., E.S.) and Bascom Palmer Eye Institute (B.L.L.), University of Miami, Miami, Florida.

Paper previously presented at: Annual Meeting of the American Society of Neuroradiology, May 20-23, 2013; San Diego, California.

This study was supported in part by the National Institutes of Health (R01NS052122).

Please address correspondence to Noam Alperin, PhD, Advanced Image Processing Laboratory, Department of Radiology, University of Miami, 1150 NW 14th St, Miami, FL 33136; e-mail: Nalperin@med.miami.edu

- Indicates open access to non-subscribers at www.ajnr.org

http://dx.doi.org/10.3174/ajnr.A3600 venous sinus occlusions. ${ }^{4}$ Posterior sclera flattening has also been observed in astronauts after long-duration exposure to microgravity. ${ }^{5,6}$ Occurrence rates of globe flattening and optic nerve protrusion in IIH and secondary $\mathrm{IH}$ are reported to be different. Work by Agid et $\mathrm{al}^{3}$ assessing several cross-sectional neuroimaging signs seen in $\mathrm{IIH}$ suggests that posterior globe flattening has the strongest diagnostic value, with specificity of $100 \%$, whereas optic nerve protrusion was not significantly associated with IIH. In contrast, Rohr et $\mathrm{al}^{4}$ assessed similar signs in patients with secondary $\mathrm{IH}$ and reported specificity of $78 \%$ for globe flattening and $100 \%$ for optic disc protrusion. Both studies, however, used subjective visual inspection to determine the presence of flattening and optic nerve protrusion.

Determining the presence and degree of globe distortions in MR imaging by visual inspection is challenging because of the $3 \mathrm{D}$ nature of the globe morphology. In addition, impression of distortion can vary with imaging orientation. 

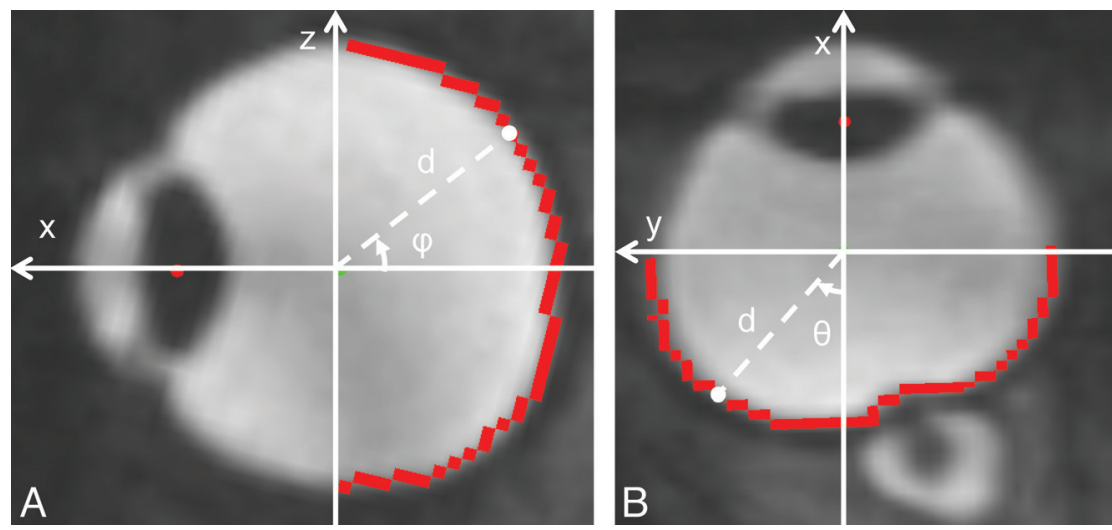

FIG 1. MR images of the globe reformatted in sagittal (A) and axial (B) planes. The coordinate system is defined by the center of mass of the lens and the globe (red and green points), respectively. The red contour marks the posterior sclera. Each point on the sclera is defined by the distance to the center of the orbit (dashed line), azimuth angle $(\theta)$, and the elevation angle $(\varphi)$.
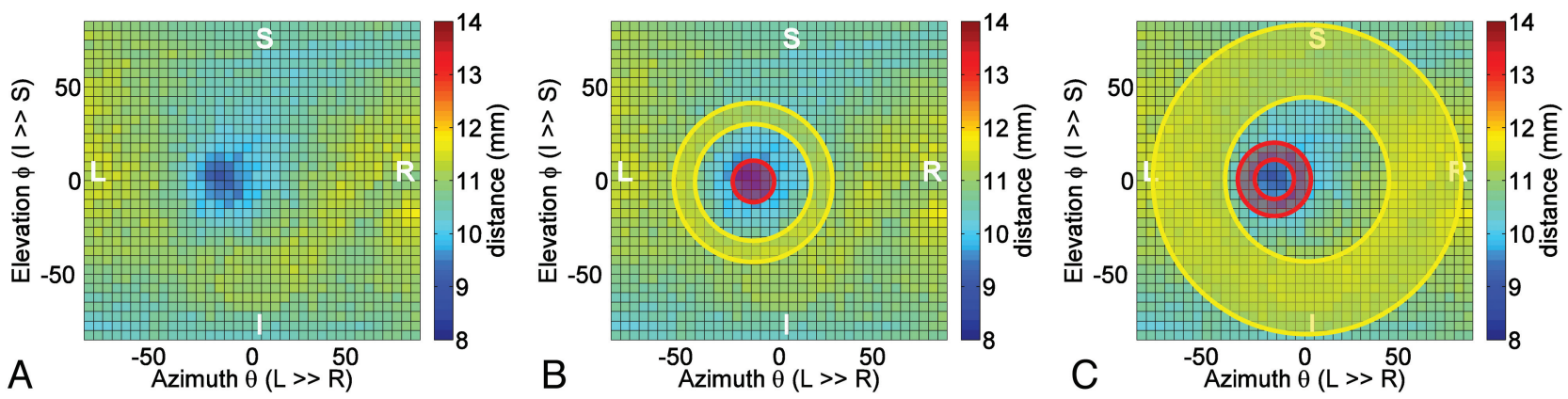

FIG 2. A 2D color-coded distance map visualizes distances between the center of the globe and points on the posterior sclera. The map represents the globe shown in Fig 1. The inward protrusion of the papilla is visualized as an off-center blue patch (A). The central (red) and peripheral (yellow) ROls used for the derivation of NP and GF are shown in $B$ and C, respectively. The angular boundaries of the central (papillar), peripappilar, and peripheral ROIs are $0-10^{\circ}, 10-18^{\circ}$, and $40-80^{\circ}$, respectively.

Flattening of the posterior sclera and inward protrusion of the optic papilla are often seen in patients with papilledema documented by fundoscopy. Papilledema, or optic nerve head edema, is caused by increased CSF volume and pressure within the optic nerve sheath leading to axoplasmic flow stasis and subsequent ischemia. ${ }^{7,8}$ The exact mechanism by which papilledema occurs is still debatable. Either mechanical or ischemic processes are currently the 2 competing theories. ${ }^{9}$ A recent comprehensive review of MR imaging of papilledema and the visual pathway lists globe flattening and optic papilla protrusion among several findings commonly seen in papilledema. ${ }^{10}$ However, because of a lack of quantitative measures of globe flattening and optic nerve protrusion, it is unknown whether papilledema grade is associated with the degrees of flattening and/or protrusion. Reliable quantification of flattening and protrusion may increase the diagnostic power of these markers and further elucidate the mechanism by which elevated intracranial pressure (ICP) causes papilledema.

An automated method for quantifying globe flattening and optic nerve protrusion is described in the present study. Measures obtained in patients with IIH and healthy control subjects were compared with subjective rating by experienced and inexperienced readers. Measurements were also tested for association with papilledema grade, intraocular pressure (IOP), and CSF opening pressure. Finally, sensitivity for detection of changes after treatment was assessed.

\section{MATERIALS AND METHODS \\ Study Participants}

All study participants provided written informed consent approved by the institutional review board. MR imaging data of 34 globes were analyzed. The data were obtained from a cohort of 7 overweight women of childbearing age $(30 \pm 12$ years; range, 17-44 years; mean body mass index, $36 \pm 4 \mathrm{~kg} / \mathrm{m}^{2}$; range, 29-41 $\mathrm{kg} / \mathrm{m}^{2}$ ) with a confirmed diagnosis of IIH as per the modified Dandy criteria ${ }^{11}$ and 6 healthy age- and weight-matched female subjects ( $30 \pm 8$ years; range, $22-44$ years; body mass index, $37 \pm 6 \mathrm{~kg} / \mathrm{m}^{2}$; range, $31-49 \mathrm{~kg} / \mathrm{m}^{2}$ ). A subcohort of 4 patients with IIH underwent a follow-up MR imaging scan approximately 2 weeks after lumbar puncture (LP) and initiation of treatment with acetazolamide.

Detailed neurologic symptoms and ophthalmologic assessments were obtained before diagnostic LP. Papilledema severity was classified by fundoscopic examination before and 2 weeks after LP, by use of the Frisen scale. ${ }^{12}$ Bilateral papilledema was found in all patients with $\mathrm{IIH}$ (mean grade, $2.0 \pm 1.0$; range, $1-4$ ). Six of 7 patients reported moderate to severe headaches and vision-related problems, including 

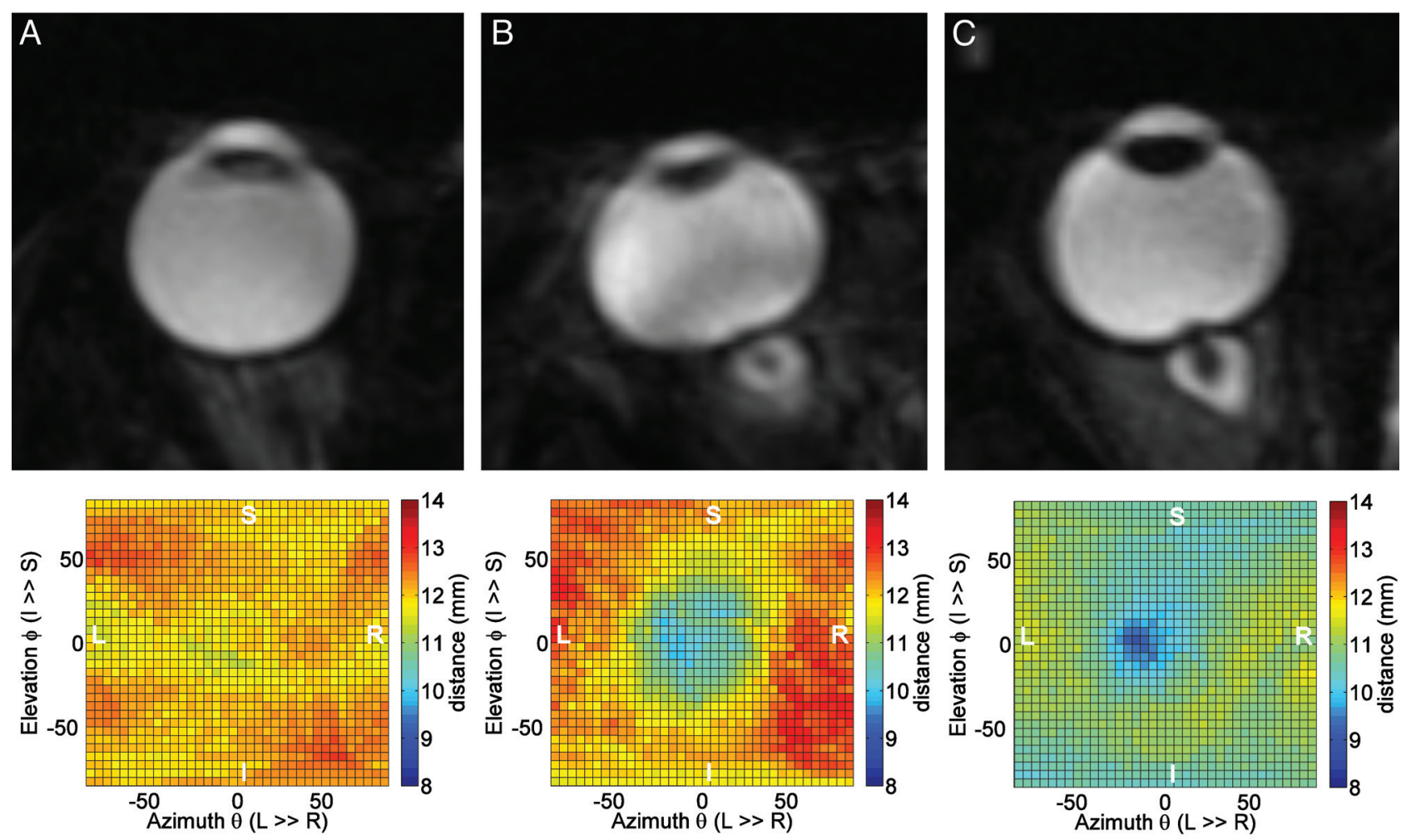

FIG 3. Reformatted MR images shown in axial plane for a normal globe (A), a flattened globe with minimal optic nerve protrusion (B), and a globe with minimal flattening and extensive optic nerve protrusion (C). Respective distance maps are shown in the bottom. The corresponding GF and NP values are $(A) 0.97$ and $0.97,(B) 0.86$ and 0.91 , and $(C) 0.91$ and 0.88 .
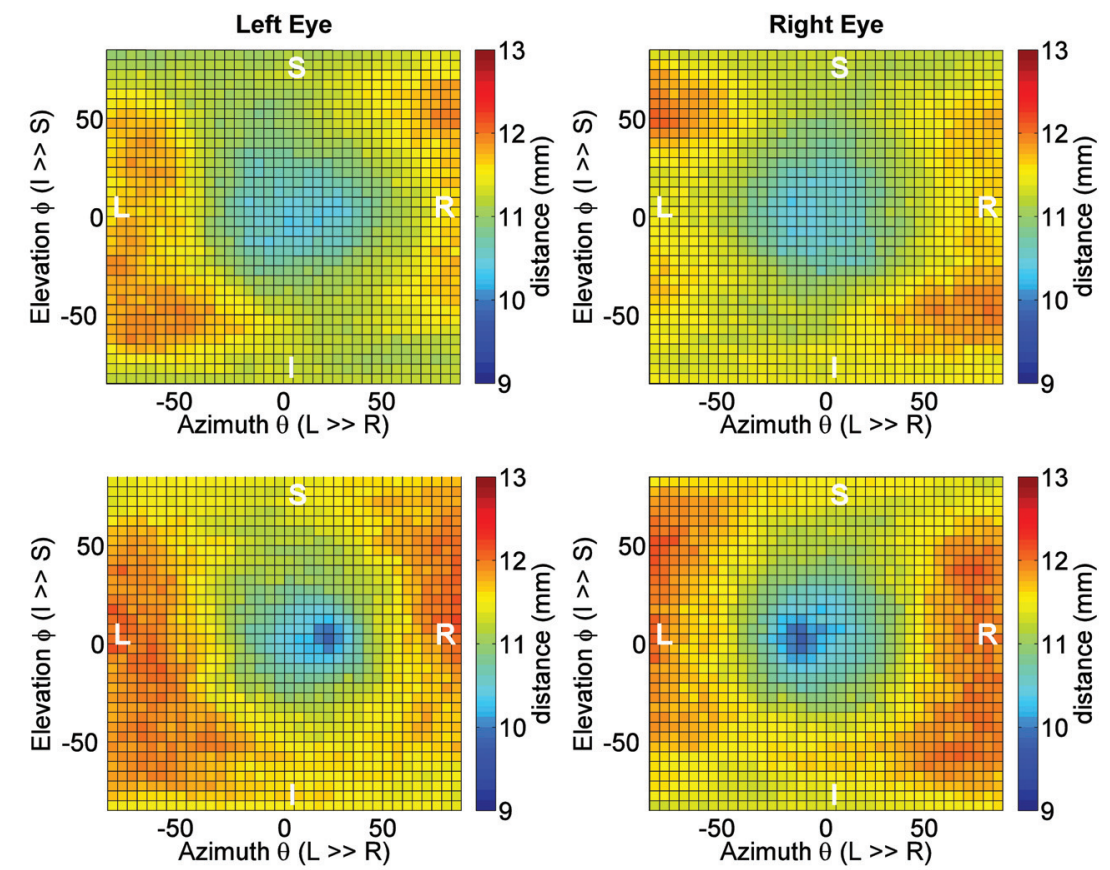

FIG 4. Average left and right eyes, 2D-distance maps obtained from the control cohort (upper row) and the IIH cohort (lower row). The presence of nerve protrusion is clearly seen in the maps from the $\mathrm{IIH}$ cohort.

blurriness, transient visual obscurations, and impaired peripheral vision. The mean opening pressure by $\mathrm{LP}$ was $36.9 \pm 7.8 \mathrm{cmH}_{2} \mathrm{O}$ (range, $26-47$ $\mathrm{cmH}_{2} \mathrm{O}$ ). IOP was measured in the $\mathrm{IIH}$ and the control cohorts, with mean values of $17.3 \pm 2.9 \mathrm{~mm} \mathrm{Hg}$ and $12.6 \pm 1.5 \mathrm{~mm} \mathrm{Hg}$, respectively.

\section{MR Imaging Scan Protocol}

MR imaging scans were performed by use of $1.5 \mathrm{~T}$ and $3 \mathrm{~T}$ scanners (Symphony and Trio; Siemens, Erlangen, Germany). Quantitative assessment of the globes was obtained from a 3D CISS sequence (A) with an isotropic resolution of 0.6 $\mathrm{mm}$ and the following parameters: TR, $6.35 \mathrm{~ms}$ (5.42 $\mathrm{ms}$ for 3T); TE, $2.82 \mathrm{~ms}$ (2.43 for $3 \mathrm{~T})$; flip angle, $47^{\circ}\left(34^{\circ}\right.$ for $\left.3 \mathrm{~T}\right)$; and bandwidth, $560 \mathrm{~Hz} /$ pixel $(650 \mathrm{~Hz} /$ pix for $3 \mathrm{~T})$. Scan time was up to $3 \mathrm{~min}-$ utes (2.5 for $3 \mathrm{~T})$.

\section{Subjective Assessment of Globe Flattening}

Flattening of the posterior ocular wall was visually evaluated by an experienced reader ( $>25$ years) and an inexperienced reader $(<1$ year $)$, blinded to the clinical diagnosis. A second reading was obtained to assess intraobserver reliability. MR images were reformatted and reviewed in both axial and sagittal views. A 4-point rating was used, in which $0=$ no flattening, $1=$ minimal flattening or deviation from circular shape at least in 1 plane, 2 = obvious flattening with apparent deviation from circular shape on both planes, and 3 = profound flattening of a large portion of the posterior wall. 


\section{Automated Measurements of Globe Deformity}

The automated method for quantifying the 3D geometry of the posterior sclera includes 3 steps: 1) segmentation, 2) identification of a reference point (center of globe), and 3) generating a 2D map of the distances from the globe center to the posterior wall. First, the MR images are aligned to a common anatomic space by use of rigid linear registration ${ }^{13}$ to ensure that both globes are located on the same axial plane. Reformatted images are then segmented by use of an expectation-maximization algorithm ${ }^{14}$ to delineate the outside boundaries of the globe and the lens. The centers of mass of the globe and the lens are then identified and are used to define an orthogonal coordinate system in which the $\mathrm{x}$ axis is the posterior-anterior orientation, or the optical axis, and the y-axis is oriented laterally from left to right, as shown in Fig 1. This framework is independent of the individual's gaze direction. Each point on the globe posterior wall is identified by use of 3 parameters: $d$, the distance in millimeters from the globe center; $\theta$, the azimuth angle (in the xy plane); and $\varphi$, the elevation angle (in the xz plane), where $\theta$ and $\varphi$ range from $-90^{\circ}$ to $+90^{\circ}$. An example of a 2D distance map is shown in Fig $2 A$. The darker shades of blue indicate shorter distances from the center and the yellow and red shades represent longer distances. A 2D map of a perfectly spherical hemisphere will have a uniform color with a distance equal to its radius.

Three measures of globe deformation are calculated by using

Table 1: Mean values of deformation measures for the control
and IIH cohorts
\begin{tabular}{cccc}
\hline Measure & Control $(\boldsymbol{n}=7)$ & $\mathrm{IIH}(\boldsymbol{n}=6)$ & $\boldsymbol{P}$ Value \\
\hline NP & $0.96 \pm 0.013$ & $0.91 \pm 0.028$ & .00002 \\
GF & $0.93 \pm 0.020$ & $0.91 \pm 0.022$ & .0035 \\
MD & $0.93 \pm 0.021$ & $0.88 \pm 0.027$ & .00002 \\
\hline
\end{tabular}

the distance map: 1) nerve protrusion (NP), depicting the extent of the nerve head protrusion; 2) globe flatness (GF), depicting the degree of flattening of the posterior wall, and 3) maximal deformation (MD), depicting the combined deformation due to the flattening and the nerve protrusion. Each measure is defined as the ratio of the mean distance within a central and a peripheral region in the $2 \mathrm{D}$ distance map. NP is obtained by use of circular and annular ROIs covering the optic nerve head and the peripapillary region, respectively. GF is obtained by use of an annular region of interest surrounding the nerve head and an annular region of interest centered at the optical axis and covering the peripheral section of the globe posterior hemisphere. The locations and extents of these regions are shown in Fig $2 B$ and $2 C$, respectively. The MD measure, which assesses the combined distortion caused by nerve protrusion and globe flattening, is obtained by use of the central ROI of NP and the peripheral ROI of GF. In a perfectly spherical globe with no protrusion, all 3 measures have a value of 1 . A lower value represents a larger inward deformation. Image analysis was implemented by use of Matlab (MathWorks, Natick, Massachusetts). Total computation time with the use of a personal computer was approximately 15 minutes.

\section{Statistical Methods}

Interrater and intrarater agreements of the readers' visual ratings were assessed by use of the $\kappa$ test. ${ }^{15}$ Association between visual rating of flatness and GF were determined by means of frequency tables and $\chi^{2}$ test, with the continuous GF discretized into 4 equally spaced intervals to match the visual rating scale. Differences between the computer-derived measures in the control and IIH cohorts were assessed by means of the unpaired $t$ test. Differences between pretreatment and posttreatment were assessed by means of the paired $t$ test. Receiver operating characteristic analysis was applied to determine thresholds that best distinguish patients with $\mathrm{IIH}$ from control subjects. Associations between papilledema grade and the derived globe deformation measures were also determined by means of $\chi^{2}$ test after discretizing of the deformation measures into 5 equally spaced intervals matching the Frisen scale. Finally, associations with IOP and opening CSF pressure during LP were tested by calculation of Pearson correlation coefficient. All statistical calculations were performed by use of MedCalc version 11.6 (MedCalc Software, Mariakerke, Belgium).

\section{RESULTS}

The highest inter rater agreement between the experienced and inexperienced readers for the visual ratings of the globe flatness was moderate, with a weighted $\kappa$ value of 0.46 . The intrarater agreement for the experienced and inexperienced readers had a similar $\kappa$ value
FIG 5. Pretreatment (upper row) and posttreatment (lower row) average distance maps of the patients with IIH who had a follow-up MR imaging scan $(n=4)$. A significant reversal of the extent of the optic nerve protrusion is visualized in the posttreatment maps. 
of 0.56 and 0.60 , respectively. The GF measure was significantly associated with the visual ratings of flatness with maximal contingency coefficients of 0.72 and 0.57 for the experienced and inexperienced readers, respectively.

Examples demonstrating the relationship between the shape of the globe and the corresponding distance map for a nearly spherical globe, a flattened globe wall with minimal protrusion, and a globe with extensive protrusion with minimal flattening are shown in Fig 3.

Average right and left distance maps obtained separately for the control and IIH cohorts are shown in Fig 4. The presence of nerve protrusion is clearly seen in both eyes of the IIH cohort but not in the control. Nerve protrusion, globe flatness, and maximal deformation measures obtained in the 2 cohorts are summarized in Table 1. These measures were all significantly larger in the control compared with IIH group: NP, $0.96+0.013$ versus $0.91 \pm$ $0.028(P=.00002) ; \mathrm{GF}, 0.93 \pm 0.020$ versus $0.91 \pm 0.022(P=$ $.003)$; and $\mathrm{MD}, 0.93 \pm 0.021$ versus $0.88 \pm 0.027(P=.00002)$, respectively.

The effect of treatment with acetazolamide on reversal of the eye globe deformation is clearly visualized in the average pretreatment and posttreatment distance maps from the subgroup of the 4 patients with IIH who underwent a follow-up MR. The pretreatment and posttreatment mean distance maps are shown in Fig 5. Pretreatment and posttreatment measures are summarized in $\mathrm{Ta}$ ble 2. Interestingly, NP but not GF was significantly larger after treatment. MD was also significantly larger because it includes the effect of NP. Pre- and post-NP values were $0.91 \pm 0.32$ and $0.94 \pm$ $0.17(P=.036)$, respectively. Pre- and post-GF values were $0.90 \pm$ 0.21 and $0.92 \pm 0.23(P=.09)$, respectively. Differences between pretreatment and posttreatment ratings by the 2 readers were not statistically significant.

The extent of nerve protrusion was significantly associated with papilledema grade, with a contingency coefficient of 0.74 and significance level of $P=.01$. In contrast, the flatness measure association with papilledema grade was not statistically significant (contingency coefficient $=0.504, P=.8$ ). Whereas the NP mea-

Table 2: Pretreatment and posttreatment mean values of deformation measures in the IIH subcohort

\begin{tabular}{cccc}
\hline Measure & IIH Pre $(\boldsymbol{n}=\mathbf{4})$ & IIH Post $(\boldsymbol{n}=\mathbf{4})$ & $\boldsymbol{P}$ Value \\
\hline NP & $0.91 \pm 0.032$ & $0.94 \pm 0.017$ & .036 \\
GF & $0.90 \pm 0.021$ & $0.92 \pm 0.023$ & .09 \\
MD & $0.88 \pm 0.024$ & $0.91 \pm 0.024$ & .011 \\
\hline
\end{tabular}
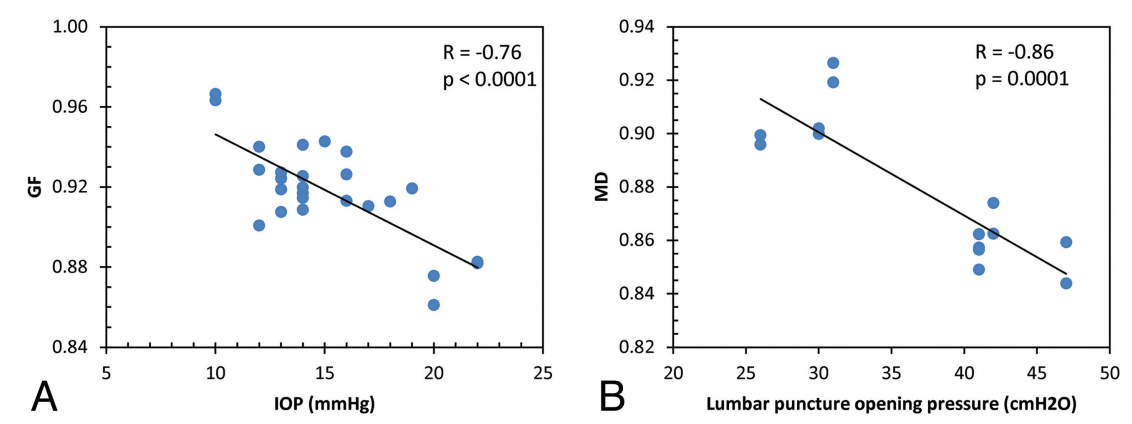

FIG 6. Scatterplots of the relationships between GF and intraocular pressure $(A)$ and between MD and CSF opening pressure (B). sure was more strongly associated with papilledema grade, the GF measure was more strongly associated with IOP. A strong negative correlation with an $R$ value of -0.76 , with $P<.0001$, was found between GF and IOP. The NP measure was also negatively correlated with IOP but with weaker association $(R=-0.54, P=$ .004). The scatterplot for GF as a function of IOP and the linear regression line are shown in Fig 6A. Finally, both NP and GF were significantly negatively correlated with the CSF opening pressure, with $R=-0.77(P=.0011)$ and $R=-0.67(P=.0089)$, respectively. As expected, the maximal deformation measure had the largest association with the CSF opening pressure, with an $R$ value of $-0.86(P=.0001)$. The scatterplot of MD with respect to the opening pressure is shown in Fig $6 B$.

\section{DISCUSSION}

Flattening of the posterior sclera and protrusion of the optic nerve head are important MR imaging findings related to visual impairment in pathologies associated with increased ICP. Determining the presence and the degree of these globe distortions by visual inspection is challenging, subjective, and strongly influenced by the orientation of the MR images. Variability and limited consistency of subjective ratings are evident from the similar moderate $\kappa$ values of interreader agreement between the experienced and the inexperienced readers and intrareader agreement of the experienced reader. The variability associated with subjective rating may partly explain reported differences in frequency of these findings in $\mathrm{IIH}$ and secondary $\mathrm{IH}^{3,4}$ Automated quantitation of the globe distortion may help to overcome the limitations associated with the subjective assessment and thereby improve diagnostic accuracy. The significant association between the subjective rating and the computer-derived GF measure supports the feasibility for a reliable automated quantitation of globe flatness.

The proposed automated method transforms the 3D globe geometry into a 2D distance map. The $2 \mathrm{D}$ computer-derived color-coded distance map provides a quantitative representation of the $3 \mathrm{D}$ morphology of the sclera in the posterior ocular hemisphere. Therefore, it has a distinctive advantage over the need for scrolling through individual MR images along different planes to gain a visual impression of the $3 \mathrm{D}$ globe deformation. Another advantage of the proposed analyses is the separate assessment of the contributions of globe flattening and nerve protrusion to the overall distortion of the globe. The reliability of the proposed computer-derived measures is supported by the statistical significance of differences in the globe distortion measures between the IIH and control cohorts, even with a relatively small number of subjects.

Whereas both the NP and the GF measures were significantly worse in the IIH cohort, the NP measure more strongly separates the patients with IIH from the healthy control subjects. Careful inspection of the distance maps of the overweight healthy cohort demonstrates some degree of globe flattening, but none had visible NP. This is also consistent with the fact that only the NP measure demonstrated a significant improvement after treatment in the 
subcohort of patients with IIH who had a follow-up scan. The NP measure was also the one that was significantly associated with the clinical papilledema grading. These findings are consistent with recent optical coherence tomography studies demonstrating inward deflection of the peripapillary retinal pigment epithelium layer in papilledema. ${ }^{16,17}$ On the basis of these observations and results, the extent of optic nerve protrusion appears to be the more clinically relevant marker for the risk of papilledema in IIH. Because larger protrusion implies larger mechanical stress around the nerve head region, it is likely that shear stress does play a role in the mechanism that leads to papilledema., ${ }^{96}$ The MR measure of NP is a potential objective measure for papilledema severity compared with the subjective Frisen scale. ${ }^{12}$

Quantitative assessment of the 2 types of globe deformation in the patients with IIH and control subjects revealed that both GF and NP were significantly correlated with IOP, with the GF measure demonstrating the stronger association. The measured IOP values in all but 1 patient with IIH were $<21 \mathrm{~mm} \mathrm{Hg}$, that is, the upper limit of normal IOP. Elevated IOP is not expected in IIH because it is not associated with impaired intraocular fluid circulation as it is in glaucoma. ${ }^{18}$ Therefore, the stronger association between IOP and GF suggests that the flattening of the posterior wall by the increased ICP probably contributes to an increase in IOP but not to the extent that is caused by impaired absorption of the aqueous humor.

Another revealing finding is the significant correlation between the deformation measures and the CSF opening pressure. This relation is expected because the primary cause for the globe deformation is increased CSF pressure transmitted to the globe through the increased CSF volume within the subarachnoid space and optic nerve sheath. ${ }^{19}$ This is consistent with the fact that MD, the combined deformation caused by NP and GF, demonstrated the strongest negative correlation with the CSF opening pressure, with an $R$ value of -0.86 .

The limitation of a small number of subjects in each cohort in the current study is somewhat lessened because both globes were assessed in each subject. Despite this, differences between patients with IIH and the healthy cohort of all 3 measures of globe deformations reached statistical significance. Furthermore, the improvement in the NP measure was statistically significant even with a subcohort size of 4 subjects. This demonstrates the reliability as well as the high sensitivity of the proposed quantitative measures of globe deformation. Furthermore, once validated in larger cohorts, the proposed analysis is suitable for clinical practice because of the relatively short scan time ( $<3$ minutes) and computation time ( $\sim 15$ minutes).

\section{CONCLUSIONS}

Assessment of ocular wall distortion on the basis of a 2D distance map provides a quantitative representation the posterior ocular geometry as well as clinically relevant measures of wall flattening and optic nerve protrusion. These measurements are likely to im- prove diagnostic accuracy and sensitivity for detection of ocular changes before and after treatment in patients with IIH.

Disclosure: Noam Alperin—RELATED: Grant: NIH*; Board Membership, Stock/Stock Options: Alperin Noninvasive Diagnostics ( ${ }^{*}$ money paid to institution).

\section{REFERENCES}

1. Brodsky MC, Vaphiades M. Magnetic resonance imaging in pseudotumor cerebri. Ophthalmology 1998;105:1686-93

2. Jinkins JR, Athale S, Xiong L, et al. MR of optic papilla protrusion in patients with high intracranial pressure. AJNR Am J Neuroradiol 1996;17:665-68

3. Agid R, Farb RI, Willinsky RA, et al. Idiopathic intracranial hypertension: the validity of cross-sectional neuroimaging signs. Neuroradiology 2006;48:521-27

4. Rohr AC, Riedel C, Fruehauf MC, et al. MR imaging findings in patients with secondary intracranial hypertension. AJNR Am J Neuroradiol 2011;32:1021-29

5. Mader TH, Gibson CR, Pass AF, et al. Optic disc edema, globe flattening, choroidal folds, and hyperopic shifts observed in astronauts after long-duration space flight. Ophthalmology 2011;118:2058-69

6. Kramer LA, Sargsyan AE, Hasan KM, et al. Orbital and intracranial effects of microgravity: findings at 3-T MR imaging. Radiology 2012;263:819-27

7. Hayreh SS. Optic disc edema in raised intracranial pressure, V: pathogenesis. Arch Ophthalmol 1977;95:1553-65

8. Tso MO, Hayreh SS. Optic disc edema in raised intracranial pressure, IV: axoplasmic transport in experimental papilledema. Arch Ophthalmol 1977;95:1458-62

9. Trobe JD. Papilledema: the vexing issues. I Neuro-ophthal 2011;31:175-86

10. Passi N, Degnan AJ, Levy LM. MR imaging of papilledema and visual pathways: effects of increased intracranial pressure and pathophysiologic mechanisms. AJNR Am J Neuroradiol 2013;34:919-24

11. Smith JL. Whence pseudotumor cerebri? J Clin Neuro-ophthal 1985;5:55-56

12. Frisen L. Swelling of the optic nerve head: a staging scheme. JNeurol Neurosurg Psychiatry 1982;45:13-18

13. Jenkinson M, Smith S. A global optimisation method for robust affine registration of brain images. Med Image Analysis 2001;5:143-56

14. Zhang Y, Brady M, Smith S. Segmentation of brain MR images through a hidden Markov random field model and the expectationmaximization algorithm. IEEE Trans Med Imaging 2001;20:45-57

15. Altman DG. Practical Statistics for Medical Research. London: Chapman \& Hall/CRC; 1991:404-08

16. Kupersmith MJ, Sibony P, Mandel G, et al. Optical coherence tomography of the swollen optic nerve head: deformation of the peripapillary retinal pigment epithelium layer in papilledema. Invest Ophthalm Vis Sci 2011;52:6558-64

17. Sibony P, Kupersmith MJ, Rohlf FJ. Shape analysis of the peripapillary RPE layer in papilledema and ischemic optic neuropathy. Invest Ophthalm Vis Sci 2011;52:7987-95

18. Dielemans I, Vingerling JR, Algra D, et al. Primary open-angle glaucoma, intraocular pressure, and systemic blood pressure in the general elderly population: the Rotterdam Study. Ophthalmology 1995;102:54-60

19. Alperin N, Ranganathan S, Bagci AM, et al. MRI evidence of impaired CSF homeostasis in obesity-associated idiopathic intracranial hypertension. AJNR Am J Neuroradiol 2013;34:29-34 